\title{
HASSE INVARIANTS FOR HILBERT MODULAR VARIETIES
}

\author{
$\mathrm{BX}$ \\ EYAL Z. GOREN \\ Department of Mathematics and Statistics, McGill University \\ 805 Sherbrooke St. West, Montreal H3A 2K6, QC, Canada \\ e-mail: goren@math.mcgill.ca
}

\begin{abstract}
Given a totally real field $L$ of degree $g$, we construct $g$ Hasse invariants on Hilbert modular varieties in characteristic $p$ and characterize their divisors. We show that these divisors give the type stratification defined by the action of $\mathcal{O}_{L}$ on the $\alpha_{p}$-elementary subgroup. Under certain conditions, involving special values of zeta functions, the product of these Hasse invariants is the reduction of an Eisenstein series of weight $p-1$.
\end{abstract}

\section{Introduction}

Moduli spaces of abelian varieties possess a rich geometric structure coming from the reflection of properties of the abelian varieties by the moduli points that parameterize them. This is even more so in positive characteristic where, beside the usual images of other modular varieties, the Frobenius morphism allows one to define several other strata. Newton, Hodge, and Ekedahl-Oort strata are particular examples. Such stratifications, besides furnishing us with (yet to be understood) elements in the Chow ring, are of importance, say, to Diophantine problems, variation of zeta functions, congruences between modular forms and special values of $L$-functions.

Usually, the definition of a stratification starts by finding a locally closed property of abelian varieties, in the sense that the set of moduli points of abelian varieties possessing this property is locally closed in the moduli space. In the case of the stratifications above this is provided by fixing the Newton polygon,

Received November 5, 1998 and in revised form December 26, 1999 
the Hodge polygon, and the isomorphism type of the $p$-torsion subgroup, respectively. As a rule, the argument that the property is locally closed runs through local deformation theory. One is thus interested in having also a global way to define such strata.

In [8] a stratification of Hilbert moduli spaces parameterizing abelian varieties with real multiplication by a totally real field $L$ in positive characteristic $p$ was defined (under the technical assumptions of principal polarization and $p$ inert in $L$ ) by means of the representation of $\mathcal{O}_{L}$ on the Dieudonné module of the $\alpha_{p}$-elementary subgroup of the abelian variety. This data is called the type of the abelian variety. The methods were of local deformation theory.

The first part of this paper documents the definition and properties of this stratification when $p$ is any unramified prime, and the polarization module is arbitrary. It may be beneficial to point out some nice properties of this stratification: It is composed of regular varieties; the chow classes can be calculated; it determines the whole $p$-torsion subgroup of an abelian variety corresponding to a geometric point on the strata as a group scheme with $\mathcal{O}_{L}$ action (though the relation between the type stratification to the Ekedahl-Oort stratification on the Siegel space is yet to be understood); the Newton polygon of the generic point of every component of a stratum is given by a simple formula. The type also turns out ([5]) to control the fibers of the forgetful morphism from the moduli space of abelian varieties with real multiplication and $\Gamma_{0}(p)$ level to the "bare" moduli space.

The second part of the paper is concerned with constructing $[L: \mathbb{Q}]$ Hilbert modular forms in characteristic $p$, denoted $H_{(j, i)}$, with the following properties: Their divisors are reduced and induce the type stratification; their $q$-expansion is one at every cusp; they factorize the Hasse-Witt determinant with respect to the $\mathcal{O}_{L}$ action. We therefore call them partial Hasse invariants. We also establish a functoriality property of these modular forms that could be formulated as well in terms of pullback of the Chern classes.

In forthcoming papers [6], [7], we show that the relations $\left(H_{(j, i)}-1\right)$ generate the kernel of the $q$-expansion map in characteristic $p$ and apply this, and geometric properties of the stratification, to prove optimal congruences for the values of $\zeta_{L}$ at negative integers, and to develop the theory of Hilbert modular forms in positive characteristic along lines similar to the elliptic case.

A particular point that merits mentioning is that the modular forms $H_{(j, i)}$ are of non-parallel weight, yet not cusp forms, and hence cannot be lifted to characteristic zero. The rest of the paper is devoted to discussing when the total 
Hasse invariant $H_{L, p}=\prod H_{(j, i)}$ (the determinant of the Hasse-Witt matrix) can be lifted to characteristic zero.

\section{A stratification of Hilbert modular varieties}

Let $L$ be a totally real field of degree $g$ over $\mathbb{Q}$, with discriminant $d_{L}$, ring of integers $\mathcal{O}_{L}$ and different ideal $\mathcal{D}_{L}=\mathcal{D}_{L / \mathbb{Q}}$. Let $C l(L)$ (resp. $\left.C l(L)^{+}\right)$denote the (resp. strict) class group of $L$, and let $h_{L}$ (resp. $h_{L}^{+}$) denote its order. The group $\mathrm{Cl}(L)^{+}$consists of classes of ideals of $L$ with their natural notion of positivity; equivalently, of projective, rank $1 \mathcal{O}_{L}$-modules $\mathcal{M}$, endowed with a notion of positivity. Let $\mathcal{M}^{+}$be the positive cone.

Let $S$ be a scheme. A HBAV over $S$ is a couple, $(A \longrightarrow S, \iota)$, consisting of an abelian scheme $A \longrightarrow S$ of relative dimension $g$ and a ring injection, $\iota: \mathcal{O}_{L} \hookrightarrow$ End $(A / S)$, such that the following holds: Let $\left(\mathcal{M}_{A}, \mathcal{M}_{A}{ }^{+}\right)$denote the module $\mathcal{M}_{A}$ of $\mathcal{O}_{L}$-linear, symmetric homomorphisms from $A$ to $A^{t}$ (the dual abelian scheme), with its natural positive cone, $\mathcal{M}_{A}{ }^{+}$, consisting of the $\mathcal{O}_{L}$-polarizations. We assume $A \otimes_{\mathcal{O}_{L}} \mathcal{M}_{A} \cong A^{t}$. We refer to [1], Section 1 for more details. If $S=\operatorname{Spec}(R)$, for some ring $R$, we shall say that $(A \longrightarrow S, \iota)$ is a HBAV over $R$.

Let $n \geq 3$. There exists a fine moduli scheme, $\mathcal{M}_{L}^{n} \longrightarrow \operatorname{Spec}\left(\mathbb{Z}\left[n^{-1}\right]\right)$, parameterizing isomorphism classes of HBAV with full level $n$ structure. It is smooth, of relative dimension $g$, over $\operatorname{Spec}\left(\mathbb{Z}\left[\left(n d_{L}\right)^{-1}\right]\right)$. Denote by $\pi: \mathfrak{X}_{L}^{n} \longrightarrow \mathcal{M}_{L}^{n}$ the universal object. We let $\mathcal{M}_{L}^{n \prime}$ be the scheme over $\operatorname{Spec}\left(\mathbb{Z}\left[n^{-1}\right]\right)$, obtained from $\mathcal{M}_{L}^{n}$ as a quotient by "forgetting the level structure". The schemes $\mathcal{M}_{L}^{n \prime}$ and $\mathcal{M}_{L}^{m \prime}$ agree over $\operatorname{Spec}\left(\mathbb{Z}\left[(m n)^{-1}\right]\right)$. We get, therefore, a scheme $\mathcal{M}_{L}$ over $\operatorname{Spec}(\mathbb{Z})$. It is a coarse moduli space for HBAV.

The scheme $\mathcal{M}_{L}$ is a disjoint union of components, $\mathcal{M}_{L}(\mathfrak{a})$, where $\mathfrak{a}$ runs over $C l(L)^{+}$. The scheme $\mathcal{M}_{L}^{n}$ is a disjoint union of components, $\mathcal{M}_{L}^{n}(\mathfrak{a}, *)$, where $\mathfrak{a}$ runs over $C l(L)^{+}$and $*$ is an additional data controlling the level $n$ structure. We have $\mathcal{M}_{L}(\mathfrak{a})(\mathbb{C}) \cong \operatorname{PGL}\left(\mathcal{O}_{L} \oplus \mathfrak{a}\right)^{+} \backslash \mathfrak{H}^{g}$, where $\mathfrak{H}$ denotes the upper half plane. More precisely, consider the lattice $\mathcal{O}_{L} \oplus \mathfrak{a}^{-1}$ in $\mathbb{R}^{2 g}$. Every $\mathcal{O}_{L}$-linear symplectic pairing on it is of the form

$$
E_{\lambda}\left(\left(x_{1}, y_{1}\right),\left(x_{2}, y_{2}\right)\right)=\operatorname{Tr}_{L / \mathbb{Q}}\left(\lambda\left(x_{1} y_{2}-x_{2} y_{1}\right)\right),
$$

for some $\lambda \in L$. It is integral iff $\lambda \in \mathfrak{a} \mathcal{D}_{L}{ }^{-1}$. Under the analytic uniformization, given by associating to $z$ in $\mathfrak{H}^{g}$ the $\mathrm{HBAV} \mathbb{C}^{g} /\left(\mathcal{O}_{L} \cdot z+\mathfrak{a}^{-1}\right), E_{\lambda}$ induces a Riemann form iff $\lambda \in \mathfrak{a} \mathcal{D}_{L}{ }^{-1}, \lambda \gg 0$. Note that under our conventions, the polarization module of $\mathcal{M}_{L}(\mathfrak{a})$ is $\left(\mathfrak{a} \mathcal{D}_{L}^{-1},\left(\mathfrak{a} \mathcal{D}_{L}^{-1}\right)^{+}\right)$.

Throughout this paper, $p$ denotes a rational prime such that $p \nmid \backslash d_{L}$. We shall denote a field of $q$ elements by $\mathbb{F}_{q}$. 
Lemma 1.1: Let $(A, \iota)$ be a HBAV over $S$. There exists $\phi \in \mathcal{M}_{A}{ }^{+}$such that $(\operatorname{deg}(\phi), p)=1$.

Proof: This follows from $A \otimes \mathcal{O}_{L} \mathcal{M}_{A} \cong A^{t}$ because $A \otimes \mathcal{O}_{L} \mathcal{M}_{A}$ represents the functor $T \mapsto A(T) \otimes \mathcal{O}_{L} \mathcal{M}_{A}$, and the morphism $A \otimes \mathcal{O}_{L} \mathcal{M}_{A} \cong A^{t}$ is determined by $\alpha \otimes \lambda \mapsto \lambda(\alpha)$ for $\alpha \in A(T), \lambda \in \mathcal{M}_{A}$ and variable scheme $T$ over $S$.

In [8], a stratification of the moduli space of principally polarized abelian schemes in characteristic $p$ with real multiplication was defined under the assumption that $p$ is inert in $L$. We indicate how to generalize that to any $p$ not dividing $d_{L}$ and to arbitrary polarization modules.

We let $\mathbb{F}$ be the minimal finite field of characteristic $p$ such that

$$
\mathcal{O}_{L} \otimes_{\mathbb{Z}} W(\mathbb{F})=\bigoplus_{\sigma \in \operatorname{Emb}\left(\mathcal{O}_{L}, W(\mathbb{F})\right)} W(\mathbb{F})
$$

where $W(\mathbb{F})$ denotes the infinite Witt vectors with coordinates in $\mathbb{F}$. Thus, if

$$
p=\mathfrak{p}_{1} \cdots \mathfrak{p}_{r}, \quad f_{i}=\operatorname{deg}\left(\mathfrak{p}_{i}\right),
$$

then $\left[\mathbb{F}: \mathbb{F}_{p}\right]=\operatorname{lcm}\left(f_{1}, \cdots, f_{r}\right)$. From now on we consider $\mathcal{M}_{L}^{n}$ as a scheme over $\operatorname{Spec}(\mathbb{F})$.

Let

$$
\left\{e_{(j, i)}: 1 \leq j \leq r, 1 \leq i \leq f_{j}\right\}
$$

be the idempotents corresponding to equation (1). Thus, in equation (1) an element $a \in \mathcal{O}_{L}$ is mapped to $\left(e_{(j, i)} a\right)$. We may also interpret the $e_{(j, i)}$ 's as $W(\mathbb{F})$-valued characters on $\mathcal{O}_{L}$, and then we denote them by $\chi_{(j, i)}$.

We write accordingly,

$$
\left\{\sigma_{(j, i)}: 1 \leq j \leq r, 1 \leq i \leq f_{j}\right\}
$$

for the embeddings of $\mathcal{O}_{L}$ into $W(\mathbb{F})$, and we assume the indexing is chosen so that

$$
\sigma \circ \sigma_{(j, i)}=\sigma_{(j, i+1)}, \quad \forall j, i,
$$

where $\sigma$ is the Frobenius homomorphism on $W(\mathbb{F})$ and, by definition, $\left(j, f_{j}+1\right)=$ $(j, 1)$. We shall abuse notation and write $e_{(j, i)}$ and $\chi_{(j, i)}$ also for the corresponding objects $\bmod p$.

Let $k \supseteq \mathbb{F}$ be a perfect field. Let $(A, \iota)$ be a HBAV over $k$. Let

$$
\alpha(A)=\operatorname{Ker}\left(\operatorname{Fr}: A \longrightarrow A^{(p)}\right) \cap \operatorname{Ker}\left(\operatorname{Ver}: A \longrightarrow A^{(1 / p)}\right) .
$$


It is a characteristic subgroup of order $p^{a}$, where $a=\operatorname{dim}_{k} \operatorname{Hom}\left(\alpha_{p}, A\right)$. Let $\mathbb{D}$ denote the contravariant Dieudonné module of $\alpha(A)$, and let us denote the indices of the characters appearing in the action of $\mathcal{O}_{L}$ on the $a$-dimensional $k$-vector space $\mathbb{D}$ by

$$
\tau(A)=\left(\tau_{1}(A), \ldots, \tau_{r}(A)\right), \quad \tau_{j}(A) \subseteq\left\{(j, i) ; 1 \leq i \leq f_{j}\right\} .
$$

That is, $\chi_{(j, i)}$ appears in the action of $\mathcal{O}_{L}$ on $\mathbb{D}$ iff $(j, i) \in \tau_{j}(A)$. We remark that by [1], Corollaire 2.9 , if $\chi_{(j, i)}$ appears, then it appears with multiplicity 1 . We call $\tau(A)$ the type of $A$.

Given a vector $\tau=\left(\tau_{1}, \ldots, \tau_{r}\right)$, where $\tau_{j} \subseteq\left\{(j, i) ; 1 \leq i \leq f_{j}\right\}$, we let $W_{\tau}$ be the minimal closed subscheme of $\mathcal{M}_{L}^{n}$ (or $\mathcal{M}_{L}$ ) such that every $(A, \iota)$ over a perfect field $k$, such that $\tau(A)$ contains $\tau$ (i.e., $\tau_{i}(A)$ contains $\tau_{i}$ for all $i$ ), is parameterized by a point of $W_{\tau}$. To ease notation, we shall write $W_{(j, i)}$ for $W_{\tau}$, when $\tau$ is the type $\left(\tau_{1}, \ldots, \tau_{r}\right)$ with $\tau_{s}=\emptyset$ if $s \neq j$ and $\tau_{j}=\{(j, i)\}$.

Given a pro-representable functor, $G$, on local artinian $k$-algebras, with $G(k)$ a singleton, we denote by $\mathcal{F} G(k)$ the corresponding formal scheme representing it. Recall that a principal quasi-polarization on a $p$-divisible group, $H$, is a symmetric isomorphism $H \longrightarrow H^{t}$. Note the following three points:

- Fix $(A, \iota)$ over $k$ with some level $n$ structure, which we shall suppress from the notation. Let $\lambda \in \mathcal{M}_{A}{ }^{+}$with $(\operatorname{deg}(\lambda), p)=1$ (Lemma 1.1). Consider the functor of local deformations of $(A, \iota)$ (resp. $(A, \iota, \lambda)$; resp. $\left.\left(A, \iota,\left(\mathcal{M}_{A}, \mathcal{M}_{A}{ }^{+}\right)\right)\right)$ into HBAV (resp. polarized HBAV; resp. HBAV with an ordered polarization module). Then the natural maps

$$
\mathcal{F}\left(A, \iota,\left(\mathcal{M}_{A}, \mathcal{M}_{A}^{+}\right)\right) \longrightarrow \mathcal{F}(A, \iota, \lambda) \longrightarrow \mathcal{F}(A, \iota)
$$

are isomorphisms.

- Let $A(p)$ be the $p$-divisible group of $A$. Let $i(p): \mathcal{O}_{L} \longrightarrow \operatorname{End}(A(p))$ and $\lambda(p)$ be the induced endomorphism structure and principal quasi-polarization on $A(p)$, respectively. Let $i(p) \otimes 1: \mathcal{O}_{L} \otimes \mathbb{Z}_{p} \longrightarrow \operatorname{End}(A(p))$ be the canonical map. Consider the functor of local deformation of $(A, \iota, \lambda)$ (resp. $(A(p), \iota(p), \lambda(p))$; resp. $(A(p), \iota(p) \otimes 1, \lambda(p)))$ into polarized HBAV (resp. principally quasi-polarized $p$ divisible groups with real multiplication by $\mathcal{O}_{L}$; resp. $p$-divisible groups with $\mathcal{O}_{L} \otimes \mathbb{Z}_{p}$ action and $\mathcal{O}_{L} \otimes \mathbb{Z}_{p}$ linear principal quasi-polarization). Then the following maps are isomorphisms:

$$
\mathcal{F}(A, \iota, \lambda) \longrightarrow \mathcal{F}(A(p), \iota(p), \lambda(p)) \longrightarrow \mathcal{F}(A(p), \iota(p) \otimes 1, \lambda(p)) .
$$


- The decomposition (2) induces a decomposition

$$
\mathcal{O}_{L} \otimes \mathbb{Z}_{p}=\bigoplus_{j=1, \ldots, r} W\left(\mathbb{F}_{p^{f_{j}}}\right)
$$

which, in turn, induces $p$-divisible groups $A_{j}$, homomorphisms

$$
\iota_{j}: W\left(\mathbb{F}_{p^{f_{j}}}\right) \longrightarrow \operatorname{End}\left(A_{j}\right),
$$

and principal quasi-polarizations $\lambda_{j}$ on $A_{j}$, such that

$$
A(p)=A_{1} \times \cdots \times A_{r}
$$

and under this isomorphism

$$
\iota(p) \otimes 1=\iota_{1} \times \cdots \times \iota_{r} ; \quad \lambda(p)=\lambda_{1} \times \cdots \lambda_{r} .
$$

We have

$$
\mathcal{F}(A(p), \iota(p), \lambda(p))=\mathcal{F}\left(A_{1}, \iota_{1}, \lambda_{\mathbf{1}}\right) \times_{\mathrm{Spec}(k)} \cdots \times_{\mathrm{Spec}(k)} \mathcal{F}\left(A_{r}, \iota_{r}, \lambda_{r}\right) .
$$

We remark that the first point can be proved using covariant Dieudonné theory by writing down explicitly the deformation matrices (see, e.g., [8] for such computations), or by crystalline techniques (see [11], Proposition 1.9).* The second point follows from the Serre-Tate Theorem, while the third is purely formal.

The deformation theory of $\left(A_{j}, \iota_{j}, \lambda_{j}\right)$ was studied in detail in [8]. It is easy now to draw the following conclusions for $\mathcal{M}_{L}^{n}$ (and with small modifications for $\left.\mathcal{M}_{L}\right)$ :

- The scheme $W_{\tau}$ is regular.

- The generic point of every component of $W_{\tau}$ has type $\tau$.

- The dimension of $W_{\tau}$ is $g-|\tau|$, where $|\tau|=\sum_{j=1}^{r}\left|\tau_{j}\right|$.

- We have $W_{\tau} \cap W_{\rho}=W_{\tau \cup \rho}$, where $\tau \cup \rho=\left(\tau_{1} \cup \rho_{1}, \ldots, \tau_{r} \cup \rho_{r}\right)$.

- The subscheme $W_{r}^{0}$, defined as

$$
W_{\tau} \backslash \bigcup_{\rho \supset \tau, \rho \neq \tau} W_{\rho}
$$

is quasi-affine.

- The components of all the $W_{\tau}$ 's have a natural structure of a $g-1$ dimensional connected colored simplicial complex.

* It requires, in fact, that $p$ be unramified in $L$. 
- Let $\mathbb{E}$ be the Hodge bundle over $\mathcal{M}_{L}^{n}$. Let

$$
W_{1}=\bigcup_{1 \leq j \leq r, 1 \leq i \leq f_{j}} W_{(j, i)}
$$

be the complement of the ordinary locus. Then, in the Chow ring,

$$
W_{1}=(p-1) \cdot c_{1}(\mathbb{E})
$$

- Let $\left(A_{1}, \iota_{1}\right),\left(A_{2}, \iota_{2}\right)$ be two HBAV's over an algebraically closed field $k$ of characteristic $p$. We have $A_{1}[p] \cong A_{2}[p]$ as groups schemes with $\mathcal{O}_{L}$-structure if and only if $\tau\left(A_{1}\right)=\tau\left(A_{2}\right)$.

- The $p$-divisible groups, up to isogeny, are precisely those of the form

$$
\bigoplus_{j=1, \ldots, r} A_{j}
$$

where $A_{j}$ is of the form $G_{\ell / f_{j}}+G_{\left(f_{j}-\ell\right) / f_{j}}$ or $f_{j} \cdot G_{1 / 2}$. The Newton polygons will be denoted accordingly: $\beta_{f_{j}, 0}<\beta_{f_{j}, 1}<\cdots$. Thus, given a HBAV over $k$, we can associate to it an $r$-tuple of Newton polygons $\left(\gamma_{1}, \ldots, \gamma_{r}\right)$; each $\gamma_{j}$ is equal to $\beta_{f_{j}, t}$ for an appropriate $t$ depending on $j$.

- Let

$$
\lambda\left(\tau_{j}\right)= \begin{cases}\left(f_{j}+1\right) / 2, & f_{j} \text { odd }, \tau_{j}=\left\{(j, 1), \ldots,\left(j, f_{j}\right)\right\} \\ \max \left\{|\rho|: \rho \subseteq \tau_{j}, \rho \text { spaced }\right\}, & \text { else }\end{cases}
$$

( $\rho$ "spaced" means $(j, i) \in \rho \Rightarrow(j, i+1) \notin \rho)$. The Newton polygon of the generic point of every component of $W_{\tau}$ is

$$
\left(\beta_{f_{1}, \lambda\left(\tau_{1}\right)}, \ldots, \beta_{f_{r}, \lambda\left(\tau_{r}\right)}\right)
$$

\section{Hasse invariants}

We formulate a condition for a prime $p>2$ and a totally real field $L$ :

$\mathbf{C}(\mathbf{L}, \mathbf{p}): \quad \zeta_{L}(2-p)$ is not $p$-integral.

We remark that by a theorem of Siegel, $\zeta_{L}(2-p)$ is a rational number. Further comments on this condition are given in Section 3 .

We recall the definition of "a modular form of weight $\chi$ ". For more details see [10], Section 1.2.

Fix a ground ring $R_{0}$, and let $\chi$ be an algebraic character of the torus $\coprod_{\mathcal{O}_{L} / \mathbb{Z}} \mathbb{G}_{m}$. A Hilbert modular form of weight $\chi$ is an $h_{L}^{+}$-tuple of modular forms $\left(f_{\mathfrak{a}}\right)_{\mathfrak{a}}$, where a runs over ideals forming a set of representatives for $C l(L)^{+}$. 
For every $\mathfrak{a}, f_{\mathfrak{a}}$ is a rule associating to a triple $(A, \iota, \omega)$ - defined over an $R_{0^{-}}$ algebra $R$ and consisting of a $\operatorname{HBAV}(A, \iota)$ over $R$ and an $\mathcal{O}_{L} \otimes R$-basis $\omega$ for $\omega_{A / R}$, such that $\left(\mathcal{M}_{A}, \mathcal{M}_{A}^{+}\right)$is in the class of $\mathfrak{a}$ - an element

$$
f_{\mathfrak{a}}(A, \iota, \omega) \in R
$$

The element $f_{\mathfrak{a}}(A, \iota, \omega)$ depends only on the $R$-isomorphism class of $(A, \iota, \omega)$ and the rule $f_{\mathfrak{a}}$ commutes with base-change. Furthermore, for every $b \in\left(\mathcal{O}_{L} \otimes R\right)^{\times}$,

$$
f_{\mathfrak{a}}(A, \iota, b \omega)=\chi(b)^{-1} f_{\mathfrak{a}}(A, \iota, \omega) .
$$

THEOREM 2.1: Let $p$ be a rational prime which is unramified at $L$.

(1) For every $(j, i)$, where $1 \leq j \leq r$ and $1 \leq i \leq f_{j}$, there exists a Hilbert modular form, $H_{(j, i)}$, over $\mathbb{F}$, of weight $\chi_{(j, i-1)}^{p} \chi_{(j, i)}^{-1}$ and level 1.* The divisor of $H_{(j, i)}$ is $W_{(j, i)}$.

(2) The $q$-expansion of $H_{(j, i)}$ at every cusp is 1.

(3) Let

$$
H_{L, p}=\prod_{j=1}^{r} \prod_{i=1}^{f_{j}} H_{(j, i)}
$$

The divisor of $H_{L, p}$ is $W_{1}$ - the complement of the ordinary locus. If $p>2$, and $p>3$ if $g=1$, and if $\mathbf{C}(\mathbf{L}, \mathbf{p})$ holds, then $H_{L, p}$ is the reduction $\bmod p$ of an Eisenstein series, $E_{L, p}$, of parallel weight $p-1$.

(4) Let $K$ be a totally real field, let $i: L \longrightarrow K$ be a field injection. Let $\mathcal{N}=$ $\operatorname{Hom}_{\mathcal{O}_{L}}\left(\mathcal{O}_{K}, \mathcal{O}_{L}\right)$. It is a left $\mathcal{O}_{L}$-module and a right $\mathcal{O}_{K}$-module. Let

$$
\Phi: \mathcal{M}_{L} \longrightarrow \mathcal{M}_{K}
$$

be the morphism defined by

$$
(A, \iota) / S \mapsto\left(A \otimes \mathcal{O}_{L} \mathcal{N}, \iota_{c a n}\right) / S
$$

where

$$
\left(A \otimes \mathcal{O}_{L} \mathcal{N}\right)(T)=A(T) \otimes_{\mathcal{O}_{L}} \mathcal{N}
$$

for any scheme $T \longrightarrow S$, and $\iota_{c a n}$ is the canonical $\mathcal{O}_{K}$ action. Then,

$$
\Phi^{*} H_{K, p}=H_{L, p}^{[K: L]} .
$$

Proof: (1) Let $(A, \iota)$ be a HBAV over $S$, where $S$ is an $\mathbb{F}$-scheme. Denote by $f: A \longrightarrow S$ the structure map. By [1], Corollaire $2.9, f_{*} \Omega_{A / S}^{1}$ is a locally free

* Note that in [5] it is stated erroneously that $H_{(j, i)}$ has weight $\chi_{(j, i)}^{p} \chi_{(j, i+1)}^{-1}$. 
$\mathcal{O}_{L} \otimes \mathcal{O}_{S}$-module of rank 1 . This implies that the usual definition of modular forms (e.g. for parallel weight, as sections of $\operatorname{det}(\mathbb{E})$ to some power) agrees with the definition above. It is therefore enough to define $H_{(j, i)}$ on quadruples $(A, t, \alpha, \omega)$, over an $\mathbb{F}$-algebra $R$, where $\alpha$ is a level $n$ structure, and $\omega$ is a generator for the free $\mathcal{O}_{L} \otimes R$ module $f_{*} \Omega_{A / R}^{1}$, where $f: A \longrightarrow \operatorname{Spec}(R)$ is the structural morphism. It will be clear that the definition does not depend on $\alpha$.

Choose an $\mathcal{O}_{L}$-linear polarization $\lambda$ on $(A, \iota)$ of degree prime to $p$ (Lemma 1.1). The pairing induced by $\lambda$,

$$
H_{d R}^{1}(A / R) \times H_{d R}^{1}(A / R) \longrightarrow R
$$

is a perfect alternating pairing. The elements,

$$
\omega_{(j, i)}=e_{(j, i)} \omega, \quad 1 \leq j \leq r, 1 \leq i \leq f_{j},
$$

form a basis for $f_{*} \Omega_{A / R}^{1}$. Let $\left\{\eta_{(j, i)}\right\}$ be the basis for $R^{1} f_{*} \mathcal{O}_{A}$ dual to the basis $\left\{\omega_{(j, i)}\right\}$. Let

$$
H_{(j, i)}(A, \iota, \alpha, \omega)=\frac{\operatorname{Fr}\left(\eta_{(j, i-1)}\right)}{\eta_{(j, i)}} .
$$

I.e., $H_{(j, i)}(A, \iota, \alpha, \omega)$ is the unique element of $R$, such that

$$
\operatorname{Fr}\left(\eta_{(j, i-1)}\right)=H_{(j, i)}(A, \iota, \alpha, \omega) \cdot \eta_{(j, i)} .
$$

Now, $H_{(j, i)}(A, \iota, \alpha, \omega)=0$ iff $(j, i) \in \tau_{j}(A)$. Indeed, if we let $\mathcal{D}=H_{d R}^{1}(A)$ denote the contravariant Dieudonné module of $A[p]$ then the Dieudonné module of $\alpha(A)$ is $\mathbb{D}=\mathcal{D} /(F \mathcal{D}+V \mathcal{D})$ and its $(j, i)$ component $\mathbb{D}_{(j, i)}$ is $\mathcal{D}_{(j, i)} /\left(F \mathcal{D}_{(j, i-1)}+\right.$ $\left.V \mathcal{D}_{(j, i+1)}\right)$. Let $\widetilde{\eta}_{(j, i-1)}$ denote any lift of $\eta_{(j, i-1)}$ to $\mathcal{D}$. Since $V \mathcal{D}_{(j, i+1)}$ is generated by $\omega_{(j, i)}$ we get that $\mathbb{D}_{(j, i)}$ is non-zero if and only if $F \mathcal{D}_{(j, i-1)}$, which is spanned by $F \widetilde{\eta}_{(j, i-1)}$, is contained in $V \mathcal{D}_{(j, i+1)}$. That is, if and only if $F \eta_{(j, i-1)}=$ 0 .

One verifies using deformation theory that $H_{(j, i)}$ vanishes to a first order along $W_{(j, i)}$ (see [8], Proof of Theorem 2.3.4, for a similar claim).

Let $s \in\left(\mathcal{O}_{L} \otimes_{\mathbb{Z}} R\right)^{\times}$. Using the decomposition,

$$
\mathcal{O}_{L} \otimes_{\mathbb{Z}} R=\left(\mathcal{O}_{L} \otimes_{\mathbb{Z}} \mathbb{F}\right) \otimes_{\mathbb{F}} R=\bigoplus_{(j, i)} R
$$

and denoting the image of $s$ by $\left(\chi_{(j, i)}(s)\right)$, we get that the basis for $f_{*} \Omega_{A / R}^{1}$ obtained from $s \omega$ is

$$
e_{(j, i)} s \omega=\chi_{(j, i)}(s) \omega_{(j, i)}, \quad 1 \leq j \leq r, 1 \leq i \leq f_{j}
$$


The dual basis is therefore

$$
\chi_{(j, i)}(s)^{-1} \eta_{(j, i)}, \quad 1 \leq j \leq r, 1 \leq i \leq f_{j}
$$

Thus, we find

$$
\frac{\operatorname{Fr}\left(\chi_{(j, i-1)}(s)^{-1} \eta_{(j, i-1)}\right)}{\chi_{(j, i)}(s)^{-1} \eta_{(j, i)}}=\left(\chi_{(j, i-1)}^{p} \chi_{(j, i)}^{-1}\right)\left(s^{-1}\right) \times \frac{\operatorname{Fr}\left(\eta_{(j, i-1)}\right)}{\eta_{(j, i)}} .
$$

That is, $H_{(j, i)}$ is a modular form of weight $\chi_{(j, i-1)}^{p} \chi_{(j, i)}^{-1}$.

Note that $H_{(j, i)}$ is independent of the polarization $\lambda$ we have chosen. Indeed, any other choice is of the form $\lambda / m$ for some $m \in L$ prime to $p$. The basis dual to $e_{(j, i)} \omega$ is then $m \eta_{(j, i)}$ and one checks that $\operatorname{Fr}\left(m \eta_{(j, i)}\right)=m \operatorname{Fr}\left(\eta_{(j, i)}\right)$.

(2) To compute the $q$-expansion of $H_{(j, i)}$, we use Katz functions as in [9], [12] - called there "generalized $p$-adic modular functions". (This "detour", though not necessary, will give us a finer result.) Recall that the domain of a Katz function is, in our case, quadruples $(A, \iota, \alpha$, triv), over an $\mathbb{F}$-algebra $R$, where $(A, \iota)$ is a HBAV over $R, \alpha$ is a level $n$ structure, and triv is an isomorphism of formal groups

$$
\text { triv } \widehat{A} \longrightarrow \widehat{\mathbb{G}_{m} / R} \otimes \mathcal{D}_{L}^{-1} \text {. }
$$

The invariant differentials on $\widehat{\mathbb{G}_{m} / R} \otimes \mathcal{D}_{L}^{-1}$ are canonically $\left(R \frac{d q}{q}\right) \otimes \mathcal{O}_{L}$, and

$$
\omega_{t r i v}=\operatorname{triv}^{*}\left(\frac{d q}{q} \otimes 1\right)
$$

is a generator for the free $\mathcal{O}_{L} \otimes R$-module $f_{*} \Omega_{A / R}^{1}$

Every modular form $f$ on $\mathcal{M}_{L}^{n}$ yields a Katz function $\hat{f}$ by the rule

$$
\hat{f}(A, \iota, \alpha, \text { triv })=f\left(A, \iota, \alpha, \omega_{t r i v}\right) .
$$

One may construct Tate objects $T_{\mathfrak{b}}$ over $\operatorname{Spec} \mathbb{Z}((\mathfrak{b} ; S))$ for every $\mathfrak{b} \in C l(L)^{+}$, which lie on the component $\mathcal{M}_{L}\left(\mathcal{D}_{L} \mathfrak{b}^{-1}\right.$ ) (see [10], Section 1.1, for the construction and notation). Recall that $T_{\mathfrak{b}}$ is constructed as the rigid analytic quotient of $\mathbb{G}_{m} \otimes \mathcal{D}_{L}{ }^{-1}$ by a lattice of periods $\underline{q}(\mathfrak{b})$, where $\underline{q}: \mathfrak{b} \longrightarrow \mathbb{G}_{m} \otimes \mathcal{D}_{L}{ }^{-1}$ is a group homomorphism. Thus, canonically,

$$
\widehat{T_{\mathfrak{b}}}=\widehat{\mathbb{G}_{m}} \otimes \mathcal{D}_{L}^{-1}
$$

The $q$-expansion at $T_{\mathfrak{b}}$ of $H_{(j, i)}$, and of the Katz function it defines, $\widehat{H_{(j, i)}}$, are the same, and both are obtained by evaluating at $T_{\mathfrak{b}}$ with its canonical trivialization. Note, therefore, that it is enough to prove that the $q$-expansion of $H_{(j, i)}$ 
for every component of $\mathcal{M}_{L}^{n}$ is 1 at one cusp. Since then $\widehat{H_{(j, i)}}$ is the constant function 1 , thus has $q$-expansion 1 at every cusp. That is why we are content to specify just one Tate object for every component. It is enough, therefore, to calculate the value of $\widehat{H_{(j, i)}}$ at $T_{\mathfrak{b}}$, and we may certainly work over the perfection, $R$, of $\mathbb{F}_{p}((\mathfrak{b} ; S))$.

Working over $R$ has the virtue that the Verschiebung morphism,

$$
\text { Ver: } f_{*} \Omega_{A / R}^{1} \longrightarrow f_{*} \Omega_{A / R}^{1},
$$

is well defined. Similarly for $H_{d R}^{1}(A / R)$.

For every $x, y \in H_{d R}^{1}(A / R)$ we have

$$
\langle\operatorname{Fr}(x), y\rangle=\langle x, \operatorname{Ver}(y)\rangle^{p} .
$$

Under the isomorphism

$$
\widehat{T_{\mathfrak{b}}}[p]=\mu_{p} \otimes \mathcal{D}_{L}^{-1},
$$

we get identifications of the tangent and cotangent spaces:

$$
\mathfrak{t}_{T_{\mathrm{b}}}=\mathfrak{t}_{\mu_{p}} \otimes \mathcal{D}_{L}{ }^{-1}, \quad \mathfrak{t}_{T_{\mathrm{b}}}^{*}=\mathfrak{t}_{\mu_{p}}^{*} \otimes \mathcal{O}_{L}=R \otimes \mathcal{O}_{L}
$$

(using the canonical differential $\frac{d q}{q}$ on $\mu_{p}=\operatorname{Spec}\left(R[q] /\left(q^{p}-1\right)\right.$ ) to identify $\mathfrak{t}_{\mu_{p}}^{*}$ with $R$ ). Under these identifications, we have

$$
\text { Ver: } R \otimes \mathcal{O}_{L} \longrightarrow R \otimes \mathcal{O}_{L}, \quad \operatorname{Ver}(r \otimes a)=r^{1 / p} \otimes a .
$$

We conclude that

$$
\operatorname{Ver}\left(e_{(j, i)} \omega_{t r i v}\right)=e_{(j, i-1)} \omega_{t r i v} .
$$

Let $\left\{\eta_{(j, i)}\right\}$ be the dual basis to $\left\{e_{(j, i)} \omega_{\text {triv }}\right\}$. Then

$$
\begin{aligned}
\widehat{H_{(j, i)}}\left(T_{\mathfrak{b}}, \iota_{c a n}, \alpha, \text { triv }\right) & =\left\langle e_{(j, i)} \omega_{t r i v}, \operatorname{Fr}\left(\eta_{(j, i-1)}\right)\right\rangle \\
& =\left\langle\operatorname{Ver}\left(e_{(j, i)} \omega_{t r i v}\right), \eta_{(j, i-1)}\right\rangle^{p} \\
& =\left\langle e_{(j, i-1)} \omega_{t r i v}, \eta_{(j, i-1)}\right\rangle^{p} \\
& =1 .
\end{aligned}
$$

(3) The assertion about the divisor of $H_{L, p}$ follows immediately from part (1). It is also immediate that $H_{L, p}$ is a modular form of parallel weight $p-1$. We proceed to construct the required Eisenstein series.

We recall the construction of an Eisenstein series $E_{\mathfrak{a}}$ on $\mathcal{M}_{L}(\mathfrak{a})(\mathbb{C})$, for every ideal class $\mathfrak{a}$ of $C l(L)^{+}$, having $p$-integral $q$-expansion congruent to $1 \quad(\bmod p)$ at one cusp of $\mathcal{M}_{L}(\mathfrak{a})(\mathbb{C})$. We then put

$$
E_{L, p}=\left(E_{\mathfrak{a}}\right)_{\mathfrak{a} \in C l(L)^{+}} .
$$


The $q$-expansion principle guarantees that $E_{L, p}$ is defined over $\mathcal{M}_{L} \times \operatorname{Spec}\left(\mathbb{Q}_{p}\right)$. Moreover, since the $q$-expansion of $H_{L, p}$ is one at every cusp, it follows that at some cusp of $\mathcal{M}_{L}(\mathfrak{a})$, for every $\mathfrak{a}, E_{L, p}(\bmod p)$ and $H_{L, p}$ have the same expansion; therefore, they are equal (see [4], Chapter X, Section 3, for properties of the $q$-expansion).

Fix $\mathfrak{a} \in C l(L)^{+}$. Let $B \in C l(L)$ and $\mathfrak{b}$ be an ideal representing $B$. Following the notation of [4], Chapter 1.6, we put

$$
G_{k, B}(z)=\mathbf{N}(\mathfrak{b})^{k} \sum_{(\alpha, \beta) \in \mathfrak{b} \mathfrak{a} \times \mathfrak{b}} \mathbf{N}(\alpha z+\beta)^{-k}
$$

where $\mathbf{N}$ denotes the norm from $L$ to $\mathbb{Q}$ and where the summation is extended over non-associated points $(\alpha, \beta) \neq(0,0)$ in $\mathfrak{b} \mathfrak{a} \times \mathfrak{b}$. We remark that $G_{k, B}$ is independent of the choice of $\mathfrak{b}$ but depends on the class $\mathfrak{a}$, which we suppress from the notation.

According to l.c., Proposition (6.4) and the remarks on p. 21 there, $G_{k, B}$ is an Eisenstein series of weight $k$ on $\operatorname{SL}\left(\mathcal{O}_{L} \oplus \mathfrak{a}\right.$ ) (in fact on $\mathrm{GL}\left(\mathcal{O}_{L} \oplus \mathfrak{a}\right)^{+}$), for $k>2$ and also for $k=2$ if $g>1$. Furthermore, its $q$-expansion at the cusp " $i \infty$ " is

$$
G_{k, B}=\frac{(2 \pi i)^{k g}}{(k-1) ! g} d_{L}^{1 / 2-k}\left(2^{-g} \zeta_{\mathcal{D}_{L} B}(1-k)+\sum \sigma_{k-1, \mathcal{D}_{L} B}\left((\nu) \mathfrak{a}^{-1} \mathcal{D}_{L}\right) e^{2 \pi i \operatorname{Tr}(\nu z)}\right)
$$

where the summation is over all totally positive $\nu \in \mathfrak{a} \mathcal{D}_{L}{ }^{-1}$, and where ${\zeta \mathcal{D}_{L} B}_{\text {is }}$ the partial zeta function of the ideal class $\mathcal{D}_{L} B$ (see 1.c. for more details). Let

$$
E_{k, \mathfrak{a}}=2^{g} \zeta_{L}(1-k)^{-1} \frac{(k-1) !^{g}}{(2 \pi i)^{k g}} \cdot d_{L}^{k-1 / 2} \cdot \sum_{B \in C l(L)} G_{k, B}
$$

The $q$-expansion of $E_{k, \mathrm{a}}$ is given by

$$
1+2^{g} \zeta_{L}(1-k)^{-1} \sum_{\nu} \sigma_{k-1}\left((\nu) \mathfrak{a}^{-1} \mathcal{D}_{L}\right) e^{2 \pi i \operatorname{Tr}(\nu z)},
$$

where the summation is over all totally positive $\nu \in \mathfrak{a} \mathcal{D}_{L}^{-1}$, and for every integral ideal $\mathfrak{b}$,

$$
\sigma_{k-1}(\mathfrak{b})=\sum_{\mathfrak{c} \mid \mathfrak{b}} \mathbf{N}(\mathfrak{c})^{k-1}
$$

(the summation is over integral ideals $\mathfrak{c}$ ). Let

$$
E_{L, p}=\left(E_{p-1, \mathfrak{a}}\right)_{\mathfrak{a}} .
$$

(4) We refer to [3] for the proof that $A \otimes \mathcal{O}_{L} \mathcal{N}$ is indeed an abelian scheme with real multiplication. One easily verifies that

$$
\mathfrak{t}_{A \otimes \mathcal{O}_{L} \mathcal{N}}=\mathfrak{t}_{A} \otimes_{\mathcal{O}_{L}} \mathcal{N} ; \quad \mathfrak{t}^{*}{ }_{A \otimes \mathcal{O}_{L}} \mathcal{N}=\mathfrak{t}_{A}^{*} \otimes_{\mathcal{O}_{L}} \mathcal{O}_{K}
$$


In particular, given a triple $(A, \iota, \omega)$ over $S$, consisting of a $\operatorname{HBAV}(A, \iota)$ and an $\mathcal{O}_{L} \otimes \mathcal{O}_{S}$-basis $\omega$ for $t_{A}^{*}$, we get a triple

$$
\left(A \otimes \mathcal{O}_{L} \mathcal{N}, \iota_{c a n}, \omega \otimes 1\right)
$$

and $\omega \otimes 1$ is an $\mathcal{O}_{K} \otimes \mathcal{O}_{S}$-basis for $t^{*}{ }_{A \otimes \mathcal{O}_{L} \mathcal{N}}$. Since it is clear from the definition of $A \otimes \mathcal{O}_{L} \mathcal{N}$ as a functor that the operation $\otimes_{\mathcal{O}_{L}} \mathcal{N}$ commutes with base-change and is functorial in $A$, we conclude that the pullback $\Phi^{*}$ of a modular form is a well defined modular form. Essentially the formula we want to prove is just the behavior of the determinant of a "linear" operator under extension of scalars. We give a more elaborate argument, though, which yields finer information (see equation (6)), even for $L=K$ and $L \longrightarrow K$ an automorphism.

We introduce the following notation. Let

$$
p=\mathfrak{p}_{1} \cdots \mathfrak{p}_{r}, \quad f_{j}=\operatorname{deg}\left(\mathfrak{p}_{j}\right)
$$

be the decomposition of $p$ in $L$. Let

$$
i\left(\mathfrak{p}_{j}\right)=\wp_{j, 1} \cdots \wp_{j, \ell_{j}} ; \quad f_{j, t}=\operatorname{deg}\left(\wp_{j, t}\right)
$$

be the decomposition of $i\left(\mathfrak{p}_{j}\right)$ in $K$. Then, the embeddings

$$
\mathcal{O}_{K} \hookrightarrow W(\mathbb{F})
$$

(F large enough) are parameterized by indices $(j, t, i)$ where $1 \leq j \leq r$, where $1 \leq t \leq \ell_{j}$ and where $1 \leq i \leq f_{j, t}$. For a fixed $(j, t)$ we may regard the indices $i$ as elements of $\mathbb{Z} / f_{j, t} \mathbb{Z}$ on which Frobenius acts by addition of 1 . Then the embeddings of $\mathcal{O}_{L}$ into $W(\mathbb{F})$ which are parameterized by $(j, s)$, where $1 \leq s \leq f_{j}$, corresponds to the cosets of the subgroup generated by $f_{j, t} / f_{j}$ in $\mathbb{Z} / f_{j, t} \mathbb{Z}$.

With this notation, the partial Hasse invariants for $K$ are denoted by $H_{(j, t, i)}$. We claim that

$$
\Phi^{*} H_{(j, t, i)}=H_{(j, i)} \text {. }
$$

Let $e_{(j, i)}$ be the idempotents for the decomposition of $\mathcal{O}_{L} \otimes W(\mathbb{F})$ and $e_{(j, t, i)}$ be those of $\mathcal{O}_{K} \otimes W(\mathbb{F})$. Under the natural inclusion

$$
\mathcal{O}_{L} \otimes W(\mathbb{F}) \hookrightarrow \mathcal{O}_{K} \otimes W(\mathbb{F})
$$

we have $e_{(j, i)}=e_{(j, 1, i)}+\cdots+e_{\left(j, \ell_{j}, i\right)}$.

Abusing notation, we write

$$
H_{(j, i)}(A, \iota, \omega)=e_{(j, i-1)}(\operatorname{Ver}(\omega) / \omega)^{p}
$$


Similarly,

$$
H_{(j, t, i)}\left(A \otimes \mathcal{O}_{L} \mathcal{N}, \iota_{c a n}, \omega \otimes 1\right)=e_{(j, t, i-1)}(\operatorname{Ver}(\omega \otimes 1) / \omega \otimes 1)^{p} .
$$

But the inclusion

$$
\mathrm{t}_{A}^{*} \hookrightarrow \mathrm{t}_{A \otimes \mathcal{O}_{L} \mathcal{N}}^{*}=\mathbf{t}_{A}^{*} \otimes \mathcal{O}_{L} \mathcal{O}_{K}
$$

is Verschiebung equivariant, and the formula (6) follows.

Corollary 2.2: We have $W_{1}=(p-1) \cdot c_{1}(\mathbb{E})$. More generally, if we let $\mathbb{E}_{(j, i)}$ denote the line bundle which is the $(j, i)$ component of the Hodge bundle and $\lambda_{(j, i)}$ the "tautological" Chern class it defines, then $W_{(j, i)}=p \lambda_{(j, i-1)}-\lambda_{(j, i)}$.

Remarks: (1) For $g=1$ the theorem reduces to a theorem of Deligne, where the condition $\mathbf{C}(\mathbb{Q}, \mathbf{p})$ follows from properties of Bernoulli numbers (see below).

(2) One may call $H_{(j, i)}$ "the partial $(j, i)$ Hasse invariant" (it is an entry of the Hasse-Witt matrix in a judiciously chosen basis) and $H_{L, p}$ "the Hasse invariant" (it is the determinant of the Hasse-Witt matrix).

\section{Remarks on condition $\mathrm{C}(\mathrm{L}, \mathrm{p})$}

We assume, to simplify notation, that $p>2$.

1. One knows that if $g k \equiv 0 \quad(\bmod p-1)$ then

$$
\operatorname{val}_{p}\left(\zeta_{L}(1-k)\right) \geq-1-\operatorname{val}_{p}(g k), \quad k>1, k \in \mathbb{N},
$$

while if $g k \not \equiv 0 \quad(\bmod p-1), \operatorname{val}_{p}\left(\zeta_{L}(1-k)\right) \geq 0$ (see [13] p. 64).* In particular,

$$
\operatorname{val}_{p}\left(\zeta_{L}(2-p)\right) \geq-1-\operatorname{val}_{p}(g) .
$$

2. Estimates for the denominator of $\zeta_{L}(1-2 m)$,

$$
w_{m}(L) \zeta_{L}(1-2 m) \in \mathbb{Z},
$$

which were conjectured by Serre and follow from the work of [2] (see [13], Section 3.7 and $[17]$ p. $73-75$ and Afterword), may suggest that often $\operatorname{val}_{p}\left(\zeta_{L}(2-p)\right) \leq-1$ (Note: $\left.p \mid w_{(p-1) / 2}\right)$.

3. Let $p \geq 3$ and prime to $d_{L}$. For $L / \mathbb{Q}$ an abelian totally real field of degree $g$ we may reformulate condition $\mathbf{C}(\mathbf{L}, \mathbf{p})$ as follows (much of what we say holds more generally): We have

$$
\zeta_{L}(s)=\prod_{\chi \in X} L(s, \chi)
$$

* See [6] for optimal congruences and bounds. 
where $X$ is the group of Dirichlet characters defining $L$. See [16], Theorem 4.3. For every $\chi$ and $n \geq 1, n \equiv 0 \quad(\bmod p-1)$, we have

$$
L_{p}(1-n, \chi)=\left(1-\chi(p) p^{n-1}\right) L(1-n, \chi) .
$$

See 1.c. p. 57 for this equality and the notation used. Furthermore, $L(\cdot, \mathbf{1})=\zeta_{\mathbb{Q}}$ and by the von-Staudt-Clausen Theorem (l.c., Theorem 5.10), we have

$$
\operatorname{val}_{p}\left(\zeta_{\mathbb{Q}}(2-p)\right)=-1
$$

(In particular $\mathbf{C}(\mathbb{Q}, \mathbf{p})$ always holds.) We deduce that

$$
\operatorname{val}_{p}\left(\zeta_{L}(2-p)\right)=-1+\sum_{\chi \neq 1} \operatorname{val}_{p}\left(L_{p}(2-p, \chi)\right)
$$

and hence

$$
\mathbf{C}(\mathbf{L}, \mathbf{p}) \text { holds } \Leftrightarrow \forall \chi \in X, \chi \neq \mathbf{1} \text {, we have } \operatorname{val}_{p}\left(L_{p}(2-p, \chi)\right)=\mathbf{0},
$$

because $L_{p}(2-p, \chi)$ is $p$-integral by 1.c., Corollary 5.13 and furthermore those values lie in an unramified extension of $\mathbb{Q}_{p}$. By the same Corollary

$$
L_{p}(2-p, \chi) \equiv L_{p}(1, \chi) \quad(\bmod p) .
$$

Therefore, $\mathbf{C}(\mathbf{L}, \mathbf{p})$ is equivalent to $L_{p}(1, \chi)$ being a $p$-adic unit for every $\chi \in X$ such that $\chi \neq 1$. Theorem 5.24 of l.c. says

$$
\prod_{\chi \in X, \chi \neq 1} L_{p}(1, \chi)=\prod_{\chi \in X, \chi \neq 1}\left(1-\frac{\chi(p)}{p}\right) \frac{2^{g-1} h_{L} R_{p}}{\sqrt{d_{L}}},
$$

where $R_{p}$ is the $p$-adic regulator of $L$. Note that $\chi(p) \neq 0$ for $p$ unramified (1.c., Corollary 3.6). Thus, for $\left(p, 2 d_{L} h_{L}\right)=1$, we have $\mathbf{C}(\mathbf{L}, \mathbf{p})$ fails if and only if $\operatorname{val}_{p}\left(R_{p}\right)>g-1$. Using Frobenius's decomposition of the determinant one can get yet more precise conditions.

In particular, for $L$ a quadratic field with fundamental unit $\epsilon$ the condition $\mathbf{C}(\mathbf{L}, \mathbf{p})$ holds if and only if $\operatorname{val}\left(\log _{p}(\epsilon)\right)=1$. As Kisilevsky pointed out, this is a "Wieferich's criterion" phenomena and by [15] (which uses the ABC conjecture) expected to happen infinitely often. In fact, one expects it to fail $1 / p$ of the times.

In the following table we give some numerical data supporting this heuristic. There are 1029 primes $\ell$ between 1 and 10,000 such that the class number of $\mathbb{Q}(\sqrt{\ell})$ is one, and there are 832 such in the range $10,000-20,000$. For every 
prime $p=3,5,7,11,13$ we give below the number of primes $\ell$ such that the condition $\mathbf{C}(\mathbb{Q}(\sqrt{\ell}), \mathbf{p})$ fails and the class number is one, in the range $1-10,000$ and $10,000-20,000$. We then let $\alpha$ and $\beta$ be the quotient of this number by the expected number $\left(\frac{1}{p} \cdot 1029\right.$ and $\frac{1}{p} \cdot 832$ respectively $)-$ up to 2 decimal places.

\begin{tabular}{|r|c|c|c|c|c|}
\hline$p$ & $1-10000$ & $\alpha$ & & $10000-20000$ & $\beta$ \\
\hline 3 & 312 & 0.91 & & 251 & 0.91 \\
5 & 196 & 0.95 & 170 & 1.02 \\
7 & 165 & 1.12 & 116 & 0.98 \\
11 & 92 & 0.98 & 67 & 0.89 \\
13 & 66 & 0.83 & 65 & 1.02 \\
\hline
\end{tabular}

We also calculated the same data for $p=11$ and $\ell$ running over the primes between 1 and 100,000 . There are 7647 such $\ell$ 's such that $\mathbb{Q}(\sqrt{\ell})$ has class number one. For $629 \ell$ 's condition $\mathbf{C}(\mathbb{Q}(\sqrt{\ell}), \mathbf{p})$ fails. The ratio between this number and the expected number $7647 / 11$ is 0.90 .

4. We observe that $\operatorname{val}_{p}\left(\zeta_{L}(1-r(p-1))\right)<0$ for $r$ a positive, prime to $p$, integer, if and only if condition $\mathbf{C}(\mathbf{L}, \mathbf{p})$ holds. Call temporarily $\operatorname{val}_{p}\left(\zeta_{L}(1-r(p-1))\right)<0$ condition $\mathbf{C}(\mathbf{L}, \mathbf{p}, \mathbf{r})$.

Using (8), (9) and that for every such $r$ one has $\operatorname{val}_{p}\left(\zeta_{\mathbb{Q}}(1-r(p-1))\right)=-1$ (as follows from the von Staudt-Clausen Theorem), we get, using an equation similar to (11), that $\mathbf{C}(\mathbf{L}, \mathbf{p}, \mathbf{r})$ holds if and only if for every non-trivial $\chi \in X$, the $p$-adic integer $L_{p}(1-r(p-1))$ is a $p$-adic unit. But by [16], Corollary 5.13, this holds if and only if $L_{p}(1, \chi)$ is a $p$-adic unit and hence is equivalent to condition $\mathbf{C}(\mathbf{L}, \mathbf{p})$.

Our interest in condition $\mathbf{C}(\mathbf{L}, \mathbf{p})$ stems from the following

Proposition 3.1: Assume that $L$ has class number one. Then, for every positive prime to $p$ integer $r$, the modular form in characteristic $p, H_{L, p}^{r}$ can be lifted to an Eisenstein series in characteristic 0 if and only if condition $\mathbf{C}(\mathbf{L}, \mathbf{p})$ holds.

Remarks: Note that for sufficiently divisible $r$, the modular form $H_{L, p}^{r}$ lifts to some modular form in characteristic zero. This follows from the ampleness of the arithmetic sheaf of modular forms.

Another observation is that for $r=p^{n} r_{0}$ we have $\operatorname{val}_{p}\left(\zeta_{\mathbb{Q}}(1-r(p-1))\right) \leq-1-n$ and hence $\operatorname{val}_{p}\left(\zeta_{L}(1-r(p-1))\right)<0$ for $n \gg 0$. For general $L$ this is Leopoldt's conjecture.

Proof: The number of cusps is equal to the class number $h_{L}$. Thus, for every $r$, $E_{r(p-1), \mathcal{D}_{L}}$ spans the one-dimensional space of modular forms of weight $r(p-1)$ on the component of the moduli space corresponding to $\mathcal{D}_{L}$. 
Using equation (4), we find

$$
E_{r(p-1), \mathcal{D}_{L}}=1+\frac{2^{g}}{\zeta_{L}(1-r(p-1))} \cdot \sum_{\nu \in \mathcal{O}_{L} ; \nu \gg 0}\left(\sum_{\mathfrak{c} \mid(\nu) ; \mathfrak{c} \subseteq \mathcal{O}_{L}} \mathbf{N}(\mathfrak{c})^{r(p-1)-1}\right) e^{2 \pi i \operatorname{Tr}(\nu z)} .
$$

Note that the coefficient of $e^{2 \pi i \operatorname{Tr}(z)}$ is $\frac{2^{g}}{\zeta_{L}(1-r(p-1))}$.

Acknowledgement: It is a pleasure to thank H. Darmon and H. Kisilevsky for enlightening remarks concerning condition $\mathbf{C}(\mathbf{L}, \mathbf{p})$, and J. Fearnley for helping me out with computer calculations done with PARI.

\section{References}

[1] P. Deligne and G. Pappas, Singularités des espaces de modules de Hilbert, en les caractéristiques divisant le discriminant, Compositio Mathematica 90 (1994), $59-74$.

[2] P. Deligne and K. Ribet, Values of Abelian $L$-functions at negative integers over totally real fields, Inventiones Mathematicae 59 (1980), 227-286.

[3] J. S. Ellenberg, Hilbert modular forms and the Galois representations associated to Hilbert-Blumenthal abelian varieties, Thesis, Harvard University, May 1998.

[4] G. van der Geer, Hilbert modular surfaces, Ergebnisse der Mathematik und ihrer Grenzgebiete, 3 Folge, Band 16 of Modern Surveys in Mathematics, SpringerVerlag, Berlin-Heidelberg, 1988.

[5] E. Z. Goren, Hilbert modular varieties in positive characteristic, in Proceedings of NATO ASI and CRM Summer School on The Arithmetic and Geometry of Algebraic Cycles, to appear, $21 \mathrm{pp}$.

[6] E. Z. Goren, Hilbert modular forms modulo $p^{m}$ - the unramified case, CICMA preprint 1998-10, submitted, $22 \mathrm{pp}$.

[7] E. Z. Goren, Hilbert modular forms modulo $p^{m}-$ the unramified case II, in preparation.

[8] E. Z. Goren and F. Oort, Stratifications of Hilbert modular varieties, Journal of Algebraic Geometry, to appear, $44 \mathrm{pp}$.

[9] N. M. Katz, p-Adic L-functions via moduli of elliptic curves, Proceedings of Symposia in Pure Mathematics 29 (1975), 479-506.

[10] N. M. Katz, $p$-Adic $L$-functions for $C M$ fields, Inventiones Mathematicae 49 (1978), 199-297.

[11] M. Rapoport, Compactifications de l'espace de modules de Hilbert-Blumenthal, Compositio Mathematica 36 (1978), 255-335. 
[12] K. A. Ribet, p-Adic interpolation via Hilbert modular forms, Proceedings of Symposia in Pure Mathematics 29 (1975), 581-592.

[13] J-P. Serre, Cohomologie des groupes discrets, Annals of Mathematics Studies 70, Princeton University Press, 1971.

[14] J-P. Serre, Formes modulaires et fonctions zêta $p$-adiques, in Proceedings of the 1972 Antwerp Summer School, Lecture Notes in Mathematics 350, SpringerVerlag, Berlin, 1973, pp. 191-268.

[15] J. H. Silverman, Wieferich's criterion and the abc-conjecture, Journal of Number Theory 30 (1988), 226-237.

[16] L. C. Washington, Introduction to Cyclotomic Fields, GTM 83, Springer-Verlag, Berlin, 1982.

[17] D. Zagier, On the values at negative integers of the zeta-function of a real quadratic field, L'Enseignement Mathématique 22 (1976), 55-95. 